\title{
Investigations of Micro-Dimples Prepared by the Multiple Sandwich-Like Electrochemical Micromachining
}

\author{
Xifang Zhang ${ }^{1, *}$, Hua $\mathrm{Li}^{1,2}$, Zhen $\mathrm{Yin}^{1}$, Ren Kun ${ }^{1}$ \\ ${ }^{1}$ College of Mechanical Engineering, Suzhou University of Science and Technology, Suzhou 215009, \\ PR China \\ ${ }^{2}$ Suzhou Key Laboratory of Precision and Efficient Manufacturing Technology, Suzhou 215009, PR \\ China \\ *E-mail: zhxflove@126.com
}

doi: $10.20964 / 2019.01 .11$

Received: 23 September 2018 / Accepted: 18 October 2018 / Published: 30 November 2018

\begin{abstract}
Micro-dimples are widely used in tribology as a surface texture and play a significant role in improving interfacial performance, allowing for reducing friction and wear, reducing vibration and noise of various mechanical components. Through-mask electrochemical micromachining (TMEMM) is a feasible method for preparing micro-dimples. However, in TMEMM, the island phenomenon and poor dimensional uniformity often occur in micro-dimples, which will weaken tribological properties. Sandwich-like electrochemical micromachining (SLEMM) could be used to fabricate micro-dimples, but these tend to be shallow and the maximum depth of $4 \mu \mathrm{m}$ for micro-dimples could be machined with the increase of machining time and applied voltage, because of the electrolytic products accumulated on the enclosed unit. In this investigation, multiple machining process of SLEMM is employed to remove the electrolytic products and generate deep micro-dimples. The experimental results showed that deep micro-dimples could be fabricated with multiple cycles of SLEMM, and at $15 \mathrm{~V}$ applied voltage and the 12th cycles process of SLEMM, the depth and diameter of micro-dimples are $18 \mu \mathrm{m}$ and $120 \mu \mathrm{m}$, respectively. In addition, numerical simulations and experimental results verified that the island phenomenon can be avoided and dimensional uniformity of micro-dimples could be enhanced by using SLEMM, compared with TMEMM.
\end{abstract}

Keywords: Micro-dimples, Through-mask electrochemical micromachining (TMEMM), Multiple, Island, Dimensional uniformity, Sandwich-like electrochemical micromachining (SLEMM)

\section{FULL TEXT}

(C) 2019 The Authors. Published by ESG (www.electrochemsci.org). This article is an open access article distributed under the terms and conditions of the Creative Commons Attribution license (http://creativecommons.org/licenses/by/4.0/). 